\title{
The influence of closure technique in total laryngectomy on the development of a pseudo-diverticulum and dysphagia
}

\author{
Martine F. van der $\operatorname{Kamp}^{1} \cdot \operatorname{Rico}$ N. P. M. Rinkel ${ }^{1} \cdot$ Simone E. J. Eerenstein $^{1}$
}

Received: 24 June 2016/ Accepted: 7 December 2016/Published online: 21 December 2016

(c) The Author(s) 2016. This article is published with open access at Springerlink.com

\begin{abstract}
Objective In total laryngectomy, the neopharynx can be closed in several ways. It is suggested that a pseudo-diverticulum is seen more frequently in patients closed with vertical closure than with " $T$ "-shaped closure, causing postoperative dysphagia. We report the results of patients treated with vertical closure and " $T$ "-shaped closure with regard to the formation of a pseudo-diverticulum and postoperative dysphagia.

Methods In our retrospective cohort study, we identified 117 consecutive laryngectomized patients treated in the VU University Medical Center of Amsterdam between March 2009 and December 2013. Evaluations with statistical analysis of postoperative outcome measures (the formation of a pseudo-diverticulum and dysphagia), qualitative and quantitative variables were conducted.

Results Patient demographics were similar between the vertical-shaped closure and the " $\mathrm{T}$ "-shaped closure groups. In $84.6 \%$ of patients with vertical closure, a pseudo-diverticulum was seen compared to $18.5 \%$ with " $\mathrm{T}$ "-shaped closure $(\mathrm{p}<0.001)$. Dysphagia was increasingly seen in patients with a pseudo-diverticulum $(60.5 \%)$ compared to patients without a pseudo-diverticulum $(39.5 \%)(\mathrm{p}=0.090)$. Conclusion Formation of a pseudo-diverticulum is more frequently seen in laryngectomy patients closed with vertical closure than in patients closed with " $T$ "-shaped closure of the neopharynx. It is favorable to implement "T"shaped closure in laryngectomy.
\end{abstract}

Paper presented at the 225th ENT association meeting, Nieuwegein, Utrecht, The Netherlands, November 20th, 2014.

Martine F. van der Kamp

martinevdkamp@hotmail.com

1 Department of Otolaryngology-Head and Neck Surgery, VU Medical Centre, Amsterdam, The Netherlands
Keywords Oncology · Head and neck surgery · Laryngectomy · Suture methods · Pseudo-diverticulum . Dysphagia

\section{Introduction}

One of the treatment options in advanced stage laryngeal and hypopharyngeal cancer is laryngectomy. In total laryngectomy, with or without pharyngectomy, the larynx and potential part of the anterior pharyngeal mucosa are removed [1]. A tracheostomy is formed and the resulting surgical defect on the anterior site of the pharynx is closed, creating the so-called neopharynx [2]. The most common surgical techniques for primary closure of the neopharynx are " $T$ "-shaped closure or vertical closure of the pharyngeal tissue. The choice of surgical technique is based on surgeons' and institute's preference. Dysphagia is one of the most common and well-known postoperative symptoms in patients after laryngectomy [3,4]. The reported incidence of postoperative dysphagia varies from 17 to $72 \%$ $[1,5,6]$. This symptom has several causes, such as tumour recurrence, pharyngeal dysmotility, stricture formation, pharyngocutaneous fistulas, and postoperative radiotherapy but also the formation of a pseudo-diverticulum [7-9]. The pseudo-diverticulum is an anteriorly located mucosalized pouch of the neopharyngeal lumen, situated at the base of the tongue (Fig. 1) [7, 10, 12]. The kind of dysphagia that it can cause can be obstructive, the feeling of having to "swallow over a hump" or with a typical regurgitative character similar to the dysphagia found in patients with a Zenker's diverticulum [10]. History of these symptoms combined with physical examination and/or barium swallow radiograph leads to the diagnosis of the pseudodiverticulum. 
Fig. 1 Barium swallow radiograph of a patient without a pseudo-diverticulum (a) and of a patient with a pseudodiverticulum (arrow) (b) after laryngectomy

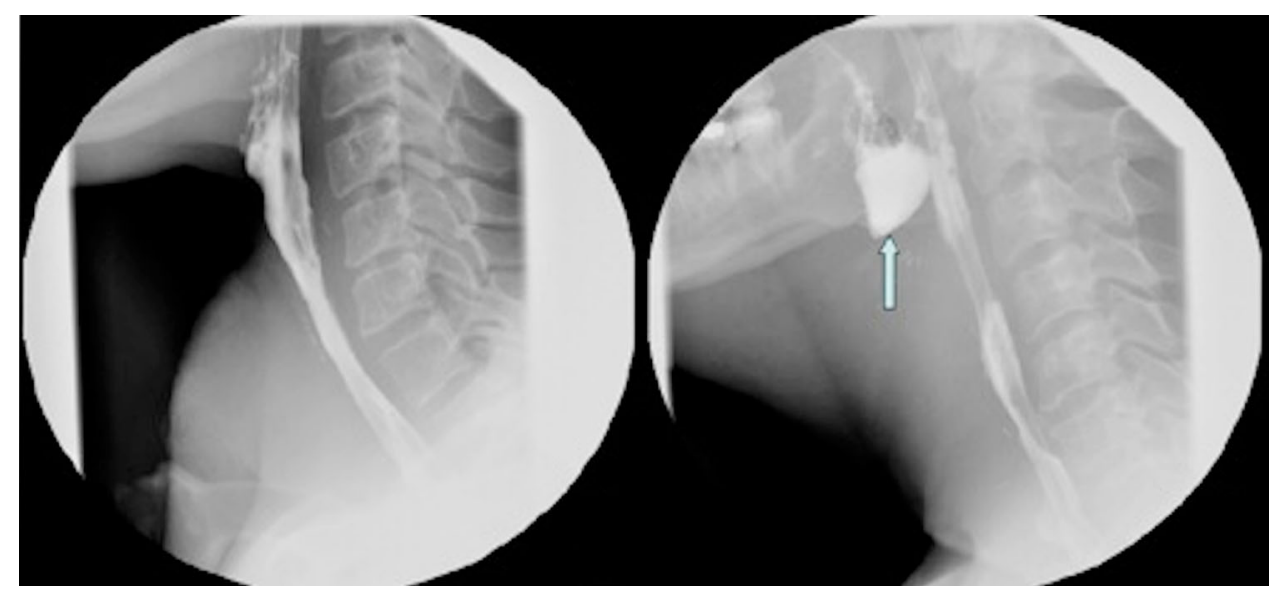

The pseudo-diverticulum might be an overlooked cause for postoperative dysphagia $[11,13]$. According to relevant literature the incidence ranges from 35 to $86 \%$ $[7-9,13,14]$. Although the possible association between closure technique in laryngectomy, pseudo-diverticulum and postoperative dysphagia has been described before, little scientific research has been published on this topic. Only one study from Davis et al. described an association between vertical closure technique and the formation of a pseudo-diverticulum in 1982 [13]. The aim of our study was to determine whether technique of surgical pharyngeal closure (vertical vs. "T"-shaped closure) is associated with the formation of a pseudo-diverticulum and whether this pseudo-diverticulum is associated with postoperative symptoms of dysphagia. We also determined if type of closure was associated with postoperative fistula formation.

\section{Materials and methods}

We conducted a retrospective cohort study in consecutive laryngectomy patients admitted to the VU University Medical Center Amsterdam between March 2009 and December 2013. Patients were excluded from analysis when they had any type of donor reconstruction of the neopharynx, for example, pectoralis major or free flap reconstruction. Also patients without postoperative barium swallow radiograph or with a barium swallow radiograph of poor quality were excluded. Postoperative follow-up ranged from seven months to five and a half years.

\section{Outcome parameters}

The primary outcome parameter was the formation of a pseudo-diverticulum. The secondary outcome parameter was postoperative dysphagia. Additionally we examined fistula formation for secondary analysis.

\section{Data}

Surgical records were examined for details about the technique of mucosal closure-vertical or " $\mathrm{T}$ "-shaped.

Information regarding postoperative dysphagia was obtained from postoperative completed validated questionnaires, the SWAL-QOL, and from the patients' medical files [15]. Dysphagia was scored as "present" when a patient's total score on the SWAL-QOL was above the cutoff point ( $\geq 14$ points) [15]. The minimum score of the SWAL-QOL is 0 points and the maximum score is 120 points. When the SWAL-QOL was not completed, dysphagia was defined as any evident swallowing dysfunction recorded in the patient's medical file. Barium swallow radiographs-made between 10 and 14 days postoperatively-from all patients were scored. Two of the authors scored the presence of a pseudo-diverticulum and measured its depth, both blinded for type of closure and dysphagia. A pseudo-diverticulum was scored when on sagittal projection an anteriorly located tissue-bar with an outpouching of the neopharynx was observed and a certain amount of contrast remained in this out-pouch after swallowing (Fig. 1). Subsequently, the deepest size of the pseudo-diverticulum was measured. Measurements in barium swallow radiographs could only be performed in units. Units were converted to millimetres by measuring the height of the third (or when not applicable, the second) cervical vertebra in units on the barium swallow radiograph and of the same vertebra in millimetres on CT-scan or MRI-scan (Fig. 2). Additionally, postoperative fistulas were identified, defined as any evident visible leakage on imaging. Medical records were used to retrieve demographical and clinical variables that may confound the association between the formation of a pseudo-diverticulum and technique of surgical closure. Variables that might cause wound-healing problems and therefore could induce the risk for developing a pseudo-diverticulum were used, 
Fig. 2 Measurement of the pseudo-diverticulum and cervical vertebra $\mathrm{C} 3$ in barium swallow radiograph after laryngopharyngectomy (a), and measurement of cervical vertebra $\mathrm{C} 3$ in $\mathrm{CT}$-scan (b)
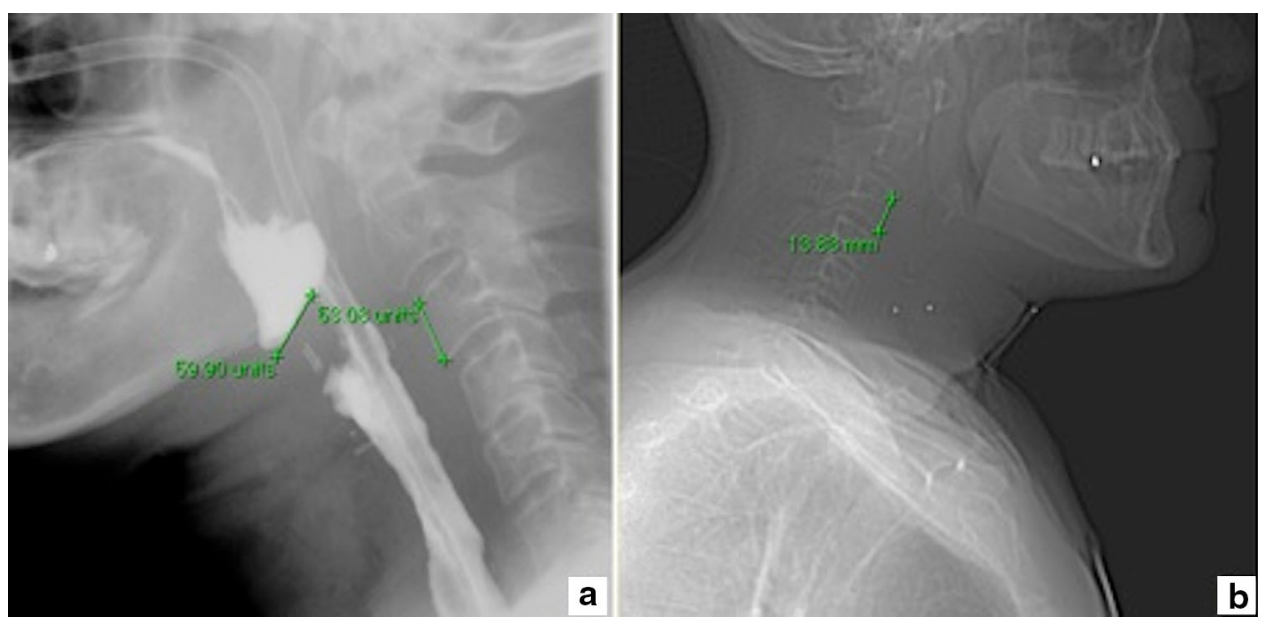

such as risk factors for head and neck squamous cell carcinomas (tobacco use and alcohol intake, tobacco use scored in pack years, and alcohol intake in units per day at time of laryngectomy) [16], adjuvant therapy (preoperative chemo- and radiotherapy), comorbidities (ASA-score) [17], postoperative complications (i.e., wound infections, fistulas, chyle leakage, stricture formation, and haemorrhage) and pre- and postoperative albumin and haemoglobin value. Other factors that could possibly induce the risk for developing a pseudo-diverticulum or dysphagia were several tumour details such as location, stage (TNM staging), primary or recurrent tumour, histopathological stage, and history of previous head- and neck carcinoma.

\section{Statistical analysis}

Statistical analyses were performed using SPSS (version 20, IBM Corp., Armonk, NY). Baseline characteristics and clinical variables were compared between the two closure methods and presented as mean and standard deviation (SD), median and range or frequencies. Independent sample $t$ test (normal data), Mann-Whitney test (non-normal data) and the Chi-square test (categorical data) were used. Variables with $p$ value $<0.05$ were considered potential confounders and further analysed via multivariable logistic regression analysis. A $p$ value $<0.05$ was considered statistically significant, a $p$ value $<0.1$ was considered to indicate a trend.

\section{Results}

\section{Patients' characteristics}

A total of 112 patients were included in our consecutive cohort of laryngectomies. For several reasons, we were obliged to exclude some patients of this certain cohort; 37 patients had a donor flap reconstruction of the neopharynx instead of primary pharyngeal closure, in five patients postoperative barium swallow radiograph was not performed or was of poor quality and in four patients the operation report was missing. This resulted in 66 patients eligible for final analysis.

Cohort information is given in Table 1. In 39 of the 66 patients, vertical closure was performed and in 27 "T"shaped closure was performed. Mean age in the vertical closure group was 64 years (SD 10.1) and in the "T"shaped closure group 61 years (SD 10.6). 34 (87.2\%) of the patients in the vertical closure group were men and 22 $(81.5 \%)$ in the "T"-shaped closure group. Timing of adjuvant therapy varied, $19(48.7 \%)$ patients in the vertical closure group underwent preoperative radiotherapy compared to $9(33.3 \%)$ in the "T"-shaped closure group. Preoperative chemotherapy was given to $5(12.8 \%)$ patients in the vertical closure group compared to $1(3.7 \%)$ patient in the " $\mathrm{T}$ "-shaped closure group. Median preoperative albumin value was $34 \mathrm{~g} / \mathrm{L}$ equal in both groups. In the vertical closure group, the median of preoperative haemoglobin was $8.3 \mathrm{mmol} / \mathrm{L}$ (range 5.2-9.7) compared to $8.6 \mathrm{mmol} / \mathrm{L}$ (range 6.6-10.3) in the "T"-shaped closure group. The vertical closure group contained $3(7.7 \%)$ hypopharyngeal and $35(89.7 \%)$ laryngeal tumours compared to $1(3.7 \%)$ hypopharyngeal tumour and $26(96.3 \%)$ laryngeal tumours in the "T"-shaped closure group. The vertical closure group showed higher numbers of patients with a primary tumour, with $19(48.7 \%)$ patients having a primary tumour and $20(51.3 \%)$ patients having a recurrent tumour. In the "T"-shaped closure $20(74.1 \%)$ patients had a primary tumour and $7(25.9 \%)$ had a recurrent tumour. In both groups, high TNM stages were seen, 24 (61.5\%) patients had T3-T4 staged tumours with $18(46.2 \%)$ patients with positive lymph nodes in the vertical closure group compared to 18 (66.7) patients with T3-T4 staged tumours and $10(37.0 \%)$ patients with positive lymph nodes in the " $\mathrm{T}$ "- 
Table 1 Patient characteristics for the two types of closure

\begin{tabular}{|c|c|c|c|}
\hline & $\begin{array}{l}\text { Vertical closure } \\
n=39\end{array}$ & $\begin{array}{l}\text { "T"-shaped closure } \\
n=27\end{array}$ & $p$ value \\
\hline Age, mean $(\mathrm{SD})$ & $64.0(10.1)$ & $60.9(10.6)$ & 0.26 \\
\hline BMI, mean (SD) & $34.3(4.4)$ & $24.9(4.8)$ & 0.37 \\
\hline Tobacco use*, median (range) & $36.5(0-110)$ & $36.8(0-120)$ & 0.70 \\
\hline Alcohol use (i.e./day), median (range) & $2.0(0-40)$ & $1.0(0-20)$ & 0.16 \\
\hline Albumin preop (g/L), median (range) & $34.0(27-63)$ & $34.0(22-42)$ & 0.39 \\
\hline $\mathrm{Hb}$ preop (millimol/L), median (range) & $8.3(5.2-9.7)$ & $8.6(6.6-10.3)$ & 0.75 \\
\hline Sex & & & 0.53 \\
\hline Male & $34(87.2 \%)$ & $22(81.5 \%)$ & \\
\hline Female & $5(12.8 \%)$ & $5(18.5 \%)$ & \\
\hline Tumour site & & & 0.64 \\
\hline Hypopharynx & $3(7.7 \%)$ & $1(3.7 \%)$ & \\
\hline Larynx & $35(89.7 \%)$ & $26(96.3 \%)$ & \\
\hline Other & $1(2.6 \%)$ & 0 & \\
\hline Histopathological & & & 0.82 \\
\hline $\mathrm{SCC}$ & $34(87.2 \%)$ & $23(85.2 \%)$ & \\
\hline Other & $5(12.8 \%)$ & $4(14.8 \%)$ & \\
\hline Type of tumour & & & 0.039 \\
\hline Primary & $19(48.7 \%)$ & $20(74.1 \%)$ & \\
\hline Recurrence & $20(51.3 \%)$ & $7(25.9 \%)$ & \\
\hline T-stage & & & 1.00 \\
\hline $\mathrm{T} 1+\mathrm{T} 2$ & $4(10.3 \%)$ & $2(7.4 \%)$ & \\
\hline $\mathrm{T} 3+\mathrm{T} 4$ & $24(61.5 \%)$ & $18(66.7 \%)$ & \\
\hline Unknown & $11(28.2 \%)$ & $7(25.9 \%)$ & \\
\hline $\mathrm{N}$-stage & & & 0.31 \\
\hline $\mathrm{N}$-positive & $18(46.2 \%)$ & $10(37.0 \%)$ & \\
\hline $\mathrm{N}$-negative & $11(28.2 \%)$ & $11(40.7 \%)$ & \\
\hline Unknown & $10(26.3 \%)$ & $6(22.2 \%)$ & \\
\hline Preop RT & & & 0.21 \\
\hline Yes & $19(48.7 \%)$ & $9(33.3 \%)$ & \\
\hline No & $20(51.3 \%)$ & $18(66.7 \%)$ & \\
\hline Preop CT & & & 0.39 \\
\hline Yes & $5(12.8 \%)$ & $1(3.7 \%)$ & \\
\hline No & $34(87.2 \%)$ & $26(96.3 \%)$ & \\
\hline Comorbidities $^{\mathrm{a}}$ & & & 0.080 \\
\hline I. No & $3(7.7 \%)$ & $1(3.7 \%)$ & \\
\hline II. Mild & $23(59.0 \%)$ & $11(40.7 \%)$ & \\
\hline III. Severe & $13(33.3 \%)$ & $15(55.6 \%)$ & \\
\hline Complications & & & 0.72 \\
\hline Yes & $22(56.4 \%)$ & $14(51.9 \%)$ & \\
\hline No & $17(43.6 \%)$ & $13(48.1 \%)$ & \\
\hline
\end{tabular}

$B M I$ body mass index, preop preoperative, $H b$ haemoglobin, $S C C$ squamous cell carcinoma, $R T$ radiotherapy, $C T$ chemotherapy

$p<0.05$ was considered significant

* Packyears

a ASA-score shaped closure group. Comorbidities were scored as I. no comorbidities, II. mild disease and III. severe disease. In the vertical closure group, respectively, 3 (7.7\%), 23
$(59.0 \%)$ and $13(33.3 \%)$ and in the "T"-shaped closure group, respectively, 1 (3.7\%), 11 (40.7\%), and 15 (55.6\%) patients were scored. In $22(56.4 \%)$ patients in the vertical 
Table 2 Results pseudodiverticulum, size, dysphagia, and fistula

\begin{tabular}{llll}
\hline & $\begin{array}{l}\text { Vertical closure } \\
n=39\end{array}$ & $\begin{array}{l}\text { "T"-shaped closure } \\
n=27\end{array}$ & $p$ value \\
\hline Pseudo-diverticulum & & & $<0.001$ \\
$\quad$ Yes & $n=33(84.6 \%)$ & $n=5(18.5 \%)$ & \\
No & $n=6(15.4 \%)$ & $12.9(5-20)$ & 0.33 \\
Size PD*, median (range) & $14.8(4-26)$ & & 0.65 \\
Dysphagia & $n=21(53.8 \%)$ & $n=13(48.1 \%)$ & 0.51 \\
Yes & $n=18(46.2 \%)$ & $n=14(51.9 \%)$ & \\
No & $n=11$ & $n=12$ & \\
SWAL-QOL total score & 32 & $21-90$ & \\
$\quad$ Mean (SD) & $7-50$ & $n=4(14.8 \%)$ & \\
Range & $n=9(23.1 \%)$ & $n=23(85.2 \%)$ & \\
Fistula & $n=30(76.9 \%)$ & & \\
Yes & & & \\
No & &
\end{tabular}

closure group varying degrees of complications were seen (i.e., fistulae, wound infections, and haemorrhage) compared to $14(51.9 \%)$ patients in the "T"-shaped group.

\section{Pseudo-diverticulum}

A pseudo-diverticulum was seen significantly more often in patients with a vertically closed neopharynx $(n=33$, $84.6 \%$ ) compared to patients with "T"-shaped closure of the neopharynx $(n=5,18.5 \% ; p<0.001)$ (Table 2). In the vertical closure group, the median size of the pseudodiverticulum was $14.8 \mathrm{~mm}$ (range 4-26 mm) compared to $12.9 \mathrm{~mm}$ (range $5-20 \mathrm{~mm}$ ) in the " $\mathrm{T}$ "-shaped closure group (Table 2). Univariate analyses with respect to closure technique identified only one potential confounder, 'Type of tumour', in the association between formation of the pseudo-diverticulum and closure technique. However, since 'Type of tumour' was not associated with the formation of a pseudo-diverticulum, it is not considered a confounder. SWAL-QOL total scores did not differ significantly between patients with or without a pseudo-diverticulum $(p=0.57)$.

\section{Dysphagia}

Twenty-three $(60.5 \%)$ patients with a pseudo-diverticulum had symptoms of dysphagia compared to 15 (39.5\%) patients without a pseudo-diverticulum. This difference was not statistically significant but indicative of a statistical trend $(p=0.088)$. No difference was found between the type of closure and postoperative dysphagia either and there was no significant difference between the SWALQOL total score in both groups $(p=0.51)$ (Table 2).

\section{Fistula}

No association was found between type of closure and postoperative fistulas. Postoperative fistulas were seen in 9 $(23.1 \%)$ of the vertically closed patients compared to 4 $(14.8 \%)$ of the "T"-shape closed patients $(p=0.40)$ (Table 2).

\section{Discussion}

Dysphagia is one of the most common and well-known symptoms seen in laryngectomized patients. One of the etiologic sources for this postoperative dysphagia is the pseudo-diverticulum. Commonly used text books such as Stell \& Maran's textbook of Head and Neck Surgery and oncology suggest that the formation of a pseudo-diverticulum is more often related to patients who have had vertical closure of the neopharynx during laryngectomy instead of " $T$ "-shaped closure [12]. However, little scientific research has been done about the influence of type of closure of the neopharynx on the development of this pseudo-diverticulum and thereby postoperative dysphagia. In the current standards, the preference of the surgeon decides what type of closure is performed during laryngectomy surgery. To determine whether there is a type of surgical closure of the neopharynx leading to the formation of a pseudo-diverticulum and postoperative dysphagia, this 
study aimed to compare two closure techniques: vertical closure versus " $\mathrm{T}$ "-shaped closure. The present study showed that the formation of a pseudo-diverticulum is more often seen in patients with vertical closure of the neopharynx than with " $\mathrm{T}$ "-shaped closure in laryngectomy. A trend $(p=0.088)$ for lower prevalence of postoperative dysphagia was reported in patients without a pseudo-diverticulum compared to patients with a pseudodiverticulum. In our opinion, the theory behind this problem is that when applying vertical closure, a surplus of tissue is formed that is creating the pseudo-diverticulum. When applying " $T$ "-shaped closure, there will be less surplus and this surplus will be fixed to the base of the tongue, which could prevent for the formation of a pseudodiverticulum. Our study showed that type of closure was not associated with the formation of fistulas or other postoperative complications. In our vision, applying " $\mathrm{T}$ "shaped closure instead of vertical closure in laryngectomy may result in reducing the risk for developing a pseudodiverticulum and probably postoperative dysphagia. In 1982, Davis et al. described an association between the type of closure and the presence and absence of a "pseudoepiglottis" (a fold of mucous membrane and sometimes scar tissue coming from the anterior pharyngeal wall into the base of the tongue, superior to the pseudo-diverticulum), in a small study with 20 patients [13]. All vertically closed patients $(n=11)$ had a pseudo-epiglottis compared to $67 \%(n=6)$ patients closed with "T"-shaped closure $(p<0.05)$. In this study, one explanation for these results was that tension on the wound edges is caused by contraction of the tongue muscles in one direction and the pharyngeal constrictors in the opposite direction. Another explanation was that after vertical closure the tension on the tongue is released, which causes the formation of the pseudo-epiglottis. In a study from Maclean et al. in 2011 with 24 included patients, the pseudo-diverticulum and a pseudo-epiglottis could not be associated with self-reported dysphagia [14]. However, in this study an association between closure technique and a pseudo-diverticulum or dysphagia was not found.

Our retrospective study has its limitations. Dysphagia could not be standardised because not all included patients filled in the SWAL-QOL questionnaire after laryngectomy. In the group of patients without a completed SWAL-QOL questionnaire, information about their swallowing function was collected from the medical files, which results in less precise measurements. Because of this, it was impossible to quantify the severity of dysphagia and therefore differences in dysphagia can be assumed. Furthermore, we did not correct for other causes of dysphagia, for instance tumour recurrence, pharyngeal dysmotility or stricture formation. This could be an explanation for the results of no statistically proved difference between the formation of a pseudo- diverticulum and postoperative dysphagia. In a future study, it would be beneficial to use information about dysphagia from validated questionnaires, so more stringent criteria could be used to define postoperative dysphagia. Furthermore, there could be disparity in the size of the measured pseudo-diverticulas. It is not possible to take barium swallow radiographs of different patients from exactly the same angles. These slightly different angles could have had influenced the measurements of the depth of the pseudo-diverticula. Strength of this study is a welldefined cohort of consecutive patients undergoing laryngectomy surgery in the VU Medical Centre. Presence and size of the pseudo-diverticulum was assessed blinded for the type of closure and the patient records were collected independently of the outcome.

\section{Conclusion}

In our retrospective study, vertical closure of the neopharynx in laryngectomy surgery showed a higher risk for developing a pseudo-diverticulum than "T"-shaped closure. Furthermore, depth of the pseudo-diverticulum was deeper in the vertical closure group compared to the "T"-shaped closure group. In the group of patients with a pseudo-diverticulum, dysphagia was more often reported than in the group of patients without a pseudo-diverticulum. Type of closure is not associated with more or particular postoperative complications. Concluding, we recommend applying " $\mathrm{T}$ "-shaped closure instead of vertical closure in laryngectomy surgery.

\section{Compliance with ethical standards}

\section{Financial disclosures None.}

Conflict of interest The authors declare that they have no conflict of interest.

Ethical approval All procedures performed in studies involving human participants were in accordance with the ethical standards of the institutional and/or national research committee and with the 1964 Helsinki declaration and its later amendments or comparable ethical standards. For this type of study formal consent is not required. This article does not contain any studies with animals performed by any of the authors.

Informed consent Informed consent was obtained from all individual participants included in the study.

Open Access This article is distributed under the terms of the Creative Commons Attribution 4.0 International License (http://crea tivecommons.org/licenses/by/4.0/), which permits unrestricted use, distribution, and reproduction in any medium, provided you give appropriate credit to the original author(s) and the source, provide a link to the Creative Commons license, and indicate if changes were made. 


\section{References}

1. Balfe DM (1990) Dysphagia after laryngeal surgery: radiologic assessment. Dysphagia 5:20-34

2. Balfe D, Koehler R, Setzen M et al (1982) Barium examination of the esophagus after total laryngectomy. Radiology 143(2):501-508

3. Ackerstaff AH, Hilgers FJM, Aaronson NK, Balm AJM (1994) Communication functional disorders and lifestyle changes after total laryngectomy. Clin Otolaryngol 19:295-300

4. Mcconnel FMS, Cerenko D, Mendelsohn MS (1988) Dysphagia after total laryngectomy. Otolaryngol Clin N Am 21:721-726

5. Ward EC, Bishop B, Frisby J, Stevens MBBS (2002) Swallowing outcomes following laryngectomy and pharyngectomy. Arch Otolaryngol Head Neck Surg 128:181-186

6. Maclean J, Cotton S, Perry A (2009) Post-laryngectomy-It's hard to swallow: an Australian study of prevalence and self-reports of swallowing function after undergoing a total laryngectomy. Dysphagia 24:172-179

7. Oursin C, Pitzer G, Fournier P, Bongartz G, Steinbrich W (1999) Anterior neopharyngeal pseudodiverticulum: A possible cause of dysphagia in laryngectomized patients. Clin Imaging 23(1):15-18

8. Nayar RC, Sharma VP, Arora M (1984) A study of the pharynx after laryngectomy. J Laryngol Otol 98(8):807-810

9. Montague ML, Davis BC, Riad M (2004) Conservative management of a large postlaryngectomy neopharyngeal diverticulum. Dysphagia 19(4):256-258

10. Jaber JJ, Greenbaum ES, Sappington JM, Burgette RC, Kramer SS, Borrowdale RW (2012) Postlaryngectomy dysphagia masking as velopharyngeal insufficiency: a simple solution for an anterior neopharyngeal diverticulum. Am J Otolaryngol 33(3):367-369

11. Kirchner JA, Scatliff JH, Dey FL, Shedd DP (1963) The pharynx after laryngectomy. Changes in its structure and function. Laryngoscope 73:18-33

12. Watkinson JC, Gilbert RW (2012) Stell \& Maran's textbook of head and neck surgery and oncology, 5th edn. Taylor \& Francis Group, Boca Raton

13. Davis RK, Vincent ME, Shapshay SM, Strong MS (1982) The anatomy and complications of " $T$ " versus vertical closure of the hypopharynx after laryngectomy. Laryngoscope 92(1):16-22

14. Maclean J, Szczesniak M, Cotton S, Cook I, Perry A (2011) Impact of a laryngectomy and surgical closure technique on swallow biomechanics and dysphagia severity. Otolaryngol Head Neck Surg 144(1):21-28

15. Rinkel RN, Verdonck-de Leeuw IM, Langendijk JA, van Reij EJ, Aaronson NK, Leemans CR (2009) The psychometric and clinical validity of the SWAL-QOL questionnaire in evaluating swallowing problems experienced by patients with oral and oropharyngeal cancer. Oral Oncol 45(8):67-87

16. Lee KW, Kuo WR, Tsai SM et al (2005) Different impact from betel quid, alcohol and cigarette: risk factors for pharyngeal and laryngeal cancer. Int J Cancer 117:831-836

17. Owens WD, Felts JA, Spitznagel Jr EL (1978) ASA physical status classifications: a study of consistency of ratings. Anesthesiology 49(4):239-243 